\title{
Compressive Fluorescence Microscopy for Biological and Hyperspectral Imaging
}

\author{
Vincent Studer $^{* \dagger}$, Jérome Bobin ${ }^{\ddagger}$, Makhlad Chahid ${ }^{*} \dagger$, S. Hamed Shams Mousavi ${ }^{*}{ }^{\dagger}$, Emmanuel Candes ${ }^{\S}$ and Maxime \\ Dahan $\pi$ \\ *Univ. Bordeaux, Interdisciplinary Institute for Neuroscience, UMR 5297, F-33000, Bordeaux, France., ${ }^{\dagger}$ CNRS, Interdisciplinary Institute for Neuroscience, UMR \\ 5297, F-33000, Bordeaux, France. ${ }^{\ddagger}$ CEA Saclay, IRFU/SEDI-Sap, ${ }^{\S}$ Departments of Mathematics, of Statistics and of Electrical Engineering, Stanford University, and \\ ` Laboratoire Kastler Brossel, CNRS UMR 8552, École Normale Supérieure, Université Pierre et Marie Curie-Paris 6
}

Submitted to Proceedings of the National Academy of Sciences of the United States of America

\begin{abstract}
The mathematical theory of compressed sensing (CS) asserts that one can acquire signals from measurements whose rate is much lower than the total bandwidth. Whereas the CS theory is now well developed, challenges concerning hardware implementations of CS-based acquisition devices-especially in optics-have only started being addressed. This paper presents an implementation of compressive sensing in fluorescence microscopy and its applications to biomedical imaging. Our CS microscope combines a dynamic structured wide-field illumination and a fast and sensitive single-point fluorescence detection to enable reconstructions of images of fluorescent beads, cells and tissues with undersampling ratios (between the number of pixels and number of measurements) up to 32 . We further demonstrate a hyperspectral mode and record images with 128 spectral channels and undersampling ratios up to 64 , illustrating the potential benefits of CS acquisition for higher dimensional signals which typically exhibits extreme redundancy. Altogether, our results emphasize the interest of CS schemes for acquisition at a significantly reduced rate and point out to some remaining challenges for CS fluorescence microscopy.
\end{abstract}

compressive sensing | sparse images | fluorescence microscopy | biological imaging

Abbreviations: CS, compressed sensing; CFM, compressive fluorescence microscopy

\section{Introduction}

$F_{p}$ uorescence microscopy is a fundamental tool in basic and applied biomedical research. Because of its optical sensitivity and molecular specificity, it is employed in an increasing number of applications which, in turn, are continuously driving the development of advanced microscopy systems that provide imaging data with ever higher spatio-temporal resolution and multiplexing capabilities. In fluorescence microscopy, one can schematically distinguish two kinds of imaging approaches, differing by their excitation and detection modalities [1]. In wide-field (WF) microscopy, a large sample area is illuminated and the emitted light is recorded on a multidetector array, such as a CCD camera. In contrast, in raster scan (RS) microscopy, a point excitation is scanned through the sample and a point detector is used to detect the fluorescence signal at each position.

While very distinct in their implementation and applications, these imaging modalities have in common that the acquisition is independent of the information content of the image. Rather, the number of measurements, either serial in RS or parallel in WF, is imposed by the Nyquist-Shannon theorem. This theorem states that the sampling frequency (namely the inverse of the image pixel size) must be twice the bandwidth of the signal, which is determined by the diffraction limit of the microscope lens equal to $\lambda / 2 \mathrm{NA}$ ( $\lambda$ is the optical wavelength and NA the objective numerical aperture). Yet, most images, including those of biological interest, can be described by a number of parameters much lower than the total number of pixels. In everyday's world, a striking consequence of this compressibility is the ability of consumer cameras with several megapixel detectors to routinely reduce the number of bits in a raw data file by an order of magnitude or two without substantial information loss. To quote from David Brady: "if it is possible to compress measured data, one might argue that too many measurements were taken" [2].

The recent mathematical theory of compressed or compressive sensing (CS - see $[3,4])$ has addressed this challenge and shown how the sensing modality could be modified to reduce the sampling rate of objects which are sparse in the sense that their information content is lower than the total bandwidth or the number of pixels suggest. The fact that one can sample such signals non-adaptively and without much information loss - if any at all-at a rate close to the image information content (instead of the total bandwidth) has important consequences, especially in applications where sensing modalities are slow or costly. To be sure, the applications of CS theory to data acquisition are rapidly growing in fields as diverse as medical resonance imaging [5, 6], analog-to-digital conversion [7, 8] or astronomy [9].

In optics, the interest in CS has been originally spurred by the demonstration of the so-called "single-pixel camera" [10]. Since then, reports have explored the potential of CS for visible and infrared imaging [11, 12], holography [13] or ghost imaging [14]. In microscopy, the feasibility of CS measurements has recently been demonstrated [15]. Altogether, these results open exciting prospects, notably for the important case of biomedical imaging. Having said this, there are very few results about the performance of CS hardware devices on relevant biological samples. As such samples often have low fluorescence, it is especially important to understand how the associated noise will affect the acquisition and reconstruction schemes.

In this paper, we describe Compressive Fluorescence Microscopy (CFM), a novel modality for fluorescence biological and hyperspectral imaging based on the concepts of CS theory. In CFM, the sample is excited with a patterned illumination and its fluorescence is collected on a point detector. Images are computationally reconstructed from measurements corresponding to a set of appropriately chosen patterns. Therefore, CFM benefits from many advantages associated with RS techniques, namely, high dynamic range, facilitated multiplexing, and wide spectral range (from the UV to the IR). In truth, the benefits of CS are particularly appealing in biology where fast, high-resolution and multicolor imaging is highly sought after.

The paper is organized as follows. We begin by recalling the principles of CS theory for optical imaging. We then turn to the de-

Reserved for Publication Footnotes 
scription of the practical implementation of CFM and of the sensing protocol. Our techniques are subsequently applied to image several relevant samples, including fluorescent beads, cultured cells and tissues. By extending our implementation, we further demonstrate the possibility of hyperspectral acquisition with up to 128 different spectral channels. A final contribution is a careful study of various noise trade-offs for CFM. We conclude the paper with a discussion of prospective CFM developments.

\section{Compressed Sensing Framework}

We wish to image a two-dimensional sample $\mathbf{x}=\{x[i]\}$, a distribution of fluorescent probes, in which $x[i]$ is the value of $\mathbf{x}$ at the pixel/location $i$ (thus one can view pixel intensities $x[i]$ as the coefficients of the image $\mathbf{x}$ in a basis of localized functions, namely, the Dirac basis). We represent this object in a basis $\mathbf{W}$ of our choosing and write

$$
\mathbf{x}=\sum_{p} c[p] \mathbf{w}_{\mathbf{p}}=\mathbf{W} \mathbf{c}
$$

where the $\mathbf{w}_{\mathbf{p}}$ 's are (orthogonal) basis functions and the $c[p]$ 's are the coefficients of $\mathbf{x}$ in the expansion. We say that the signal is $K$-sparse if at most $K$ of these coefficients are nonzero. An empirical fact is that most images of interest are well approximated by $K$-sparse expansions with $K$ much less than the number of pixels $N$, and this is the reason why data compression is effective; one can store and transmit quantizations of the large coefficients, ignore the small ones, and suffer little distortion.

In our imaging setup, we measure correlations between the image of interest $\mathbf{x}$ and sensing waveforms $\phi_{\mathbf{k}}$ taken from another basis $\boldsymbol{\Phi}$; that is, we measure

$$
y_{k}=\left\langle\mathbf{x}, \phi_{\mathbf{k}}\right\rangle=\sum_{i} x[i] \phi_{k}[i] .
$$

Here, $\phi_{\mathbf{k}}$ is an illumination or intensity pattern so that $y_{k}$ is obtained by collecting all the fluorescence corresponding to those pixels that have been illuminated on a single-point detector. Wide-field and point-like excitation are two extreme cases, corresponding respectively to a uniform sensing waveform $\left(\phi_{k}[i]=1\right.$ for all $\left.i\right)$ and to a spike or Dirac waveform.

In its simplest form, CS theory asserts that if the signal $\mathrm{x}$ is sparse in the representation $\mathbf{W}$, then only few measurements of the form [1] are sufficient for perfect recovery provided the sensing and representation waveforms, respectively $\phi_{\mathbf{k}}$ and $\mathbf{w}_{\mathbf{p}}$, are incoherent $[4,16]$. Two systems are said to be incoherent if any element in one of the two cannot be expressed as a sparse linear combination of elements taken from the other. Formally, the coherence between two orthobases $\mathbf{W}$ and $\boldsymbol{\Phi}$ of $\mathbb{R}^{N}$ is measured by the parameter $\mu(\mathbf{W}, \boldsymbol{\Phi})$ ranging between 1 and $N$ :

$$
\mu(\mathbf{W}, \boldsymbol{\Phi})=N \max _{p, k}\left|\left\langle\mathbf{w}_{\mathbf{p}}, \phi_{\mathbf{k}}\right\rangle\right|^{2} .
$$

The Fourier and Dirac bases are in this sense maximally incoherent (we need many spikes to synthesize a sinusoid and vice versa) and $\mu=1$. On the opposite, two identical bases are maximally coherent and, in this case, $\mu=N$. Hence, incoherence expresses the idea of the level of dissimilarity between any two representations of a signal. With this in mind, one perceives how each incoherent measurement - a projection on an element of the basis $\boldsymbol{\Phi}$-conveys a little bit of information about all the entries of the coefficient vector c. An important result in CS theory states that $K$-sparse signals can be recovered exactly from comparably few measurements in an incoherent system. Further, recovery is achieved by solving a tractable optimization program - a linear program. One solves

$$
\min _{\mathbf{c} \in \mathbb{R}^{N}}\|\mathbf{c}\|_{\ell_{1}} \text { subject to } y_{k}=\left\langle\phi_{\mathbf{k}}, \mathbf{W} \mathbf{c}\right\rangle \text {, for all } k=1 \ldots M \text {. }
$$

When $M$ measurements are chosen uniformly at random from the basis $\boldsymbol{\Phi}$, the recovery is exact with very high probability; that is, the solution sequence $\hat{\mathbf{c}}$ obeys $\hat{\mathbf{x}}=\sum_{p} \hat{c}[p] \mathbf{w}_{\mathbf{p}}=\mathbf{x}$, provided that

$$
M \geq C \mu(\mathbf{\Phi}, \mathbf{W}) K \log N,
$$

where $C$ is a constant on the order of unity. This result emphasizes both the role of the coherence and the potential gain for large images due to the logarithmic dependence in the pixel size. For incoherent pairs, we only need on the order of $K \log N$ random samples.

We have discussed sparse signals above for ease of exposition. However, the theory extends to approximately sparse signals and to noisy data. For instance, if the signal is well approximated by a $K$ sparse signal (some would say that it is compressible), then the reconstruction error is shown to be small. Further, the recovery is not sensitive to noise in the sense that the error degrades gracefully as the signal-to-noise ratio decreases. We refer to [17] and references therein for quantitative statements.

\section{Compressive Fluorescence Microscopy: Implementation}

Experimental setup. Our setup is based on a standard epifluorescence inverted microscope (Nikon Ti-E) as shown in Figure A. To generate spatially modulated excitation patterns, we incorporated a Digital Micromirror Device (DMD) in a conjugate image plane of the excitation path. The DMD is a 1024-by-768 array of micromirrors (Texas-Instrument Discovery 4100, Vialux, Germany) of size $13.68 \times 13.68 \mu \mathrm{m}$ each, and which can be shifted between two positions oriented at $+12^{\circ}$ or $-12^{\circ}$ with respect to the DMD surface. They are all independently configurable at frequency up to $20 \mathrm{kHz}$. The DMD is carefully positioned so that the optical axis (defined by the
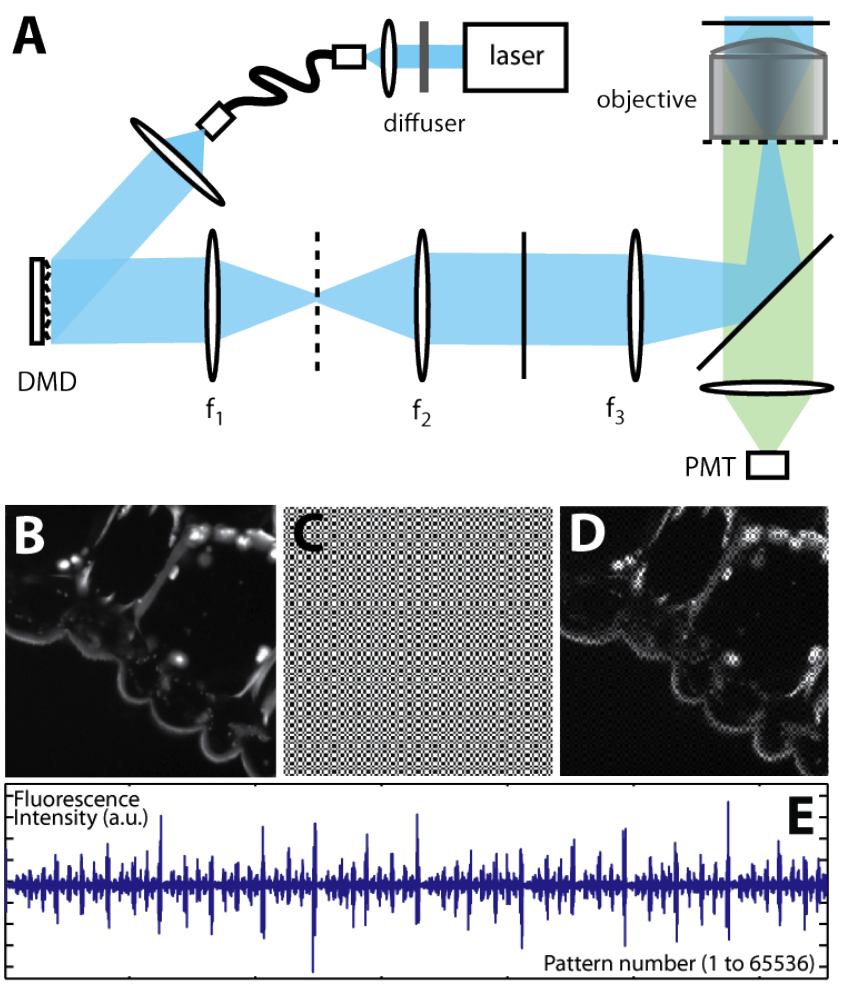

Fig. 1. (A) Experimental set-up. The dotted and plain segments correspond to planes respectively conjugated to the pupil and sample planes. (B) Slice of lily anther (endogenous fluorescence with epifluorescence microscopy image recorded on a CCD camera). (C) Projection of a Hadamard pattern on a uniform fluorescent sample. (D) Projection of the same Hadamard pattern on the biological sample. (E) Fluorescence intensity during an acquisition sequence. 
microscope lens and the dichroic mirror DM (Figure A)) is orthogonal to the plane of the DMD.

As light source, we used a laser bench (Roper Scientific, France) equipped with two superimposed continuous-wave laser $(\lambda=488 \mathrm{~nm}$, Coherent, USA and $\lambda=561 \mathrm{~nm}$, Cobolt, Sweden). The laser beam first passed through a rotating diffuser or a phase scrambler (Dyoptika, Ireland) in order to reduce the spatial coherence and was then coupled to a $200 \mu \mathrm{m}$ multimode fiber. At the fiber output, the laser beam was expanded into a $2 \mathrm{~cm}$ diameter collimated beam. This beam was oriented towards the DMD at an angle of incidence corresponding to twice the tilting angle of the DMD mirrors (approx. $24^{\circ}$ ); a micromirror oriented at $+12^{\circ}$ would reflect the light into the microscope and appear as a bright pixel in the sample plane and, inversely, micromirrors oriented at $-12^{\circ}$ appear as dark pixels. Depending on the samples, we used an air (Nikon, 20X, Plan Apo VC NA 0.75) or an oil-immersion objective (Nikon, 60X Plan Apo Tirf, NA 1.45). When the 20x air lens was used, the imaging lenses (lenses $f_{1}, f_{2}, f_{3}$ in Figure A) were chosen to introduce a $1.5 \mathrm{X}$ reduction. The overall magnification of the image of the DMD on the sample was $1 / 30$ and the size of a single micromirror equal to $456 \mathrm{~nm}$. When the $60 \mathrm{x}$ lens was used, a different set of imaging lenses was chosen which only served as a 1x relay; here, the image size of a single micromirror in the sample plane was equal to $228 \mathrm{~nm}$. Upon illumination with an intensity pattern (excitation intensity $\sim 20$ to $60 \mathrm{~W} / \mathrm{cm}^{2}$ ), the sample fluorescence was detected on a photomultiplier tube PMT (Hamamatsu) and sampled at $96 \mathrm{kHz}$ using an analog-digital converter board (PCI-4462, National Instruments, USA) (Figure B-D). In CS measurements, the information on the sample is thus contained in the variations of the intensity signal as a function of the illumination pattern (Figure E). The WF image of the sample could also be directly formed on a camera (ImagEM, Hamamatsu, Japan) placed on the output port of the microscope. For hyperspectral imaging, the PMT was replaced by a fast and sensitive spectral detector described later in the paper. Note that the role of the DMD in our set-up differs from that in the "single-pixel camera" [10] or in some other microscopy setups [15]. In the latter, the modulator is placed between the sample and the detector, meaning that it is used to select some of the light within the total signal, rather than to control the excitation pattern. Our choice is motivated by the low level of fluorescence encountered in biological samples such as living cells labelled with fluorescent proteins. Indeed, the overall efficiency of a DMD is $68 \%$ and falls down to $34 \%$ when only one half of the mirrors are tilted. In our case, the photon collection efficiency is only limited by the numerical aperture of the microscope lens and the quantum yield of the detector as in conventional epifluorescence microscopy.

Choice of the illumination patterns. For the practical implementation of a CS-based image acquisition system, it is essential to determine which incoherent basis should be used when no prior information on the signal is available. There are measurement ensembles, such as the partial Fourier or Hadamard systems, known to be highly incoherent with the bases in which most natural images are sparse. When excitation patterns are generated by micromirrors, $\phi_{k}[i]$ is a binary waveform taking on the two values 0 or 1 . An appealing choice for $\boldsymbol{\Phi}$ is then the Hadamard system known to be incoherent with the Dirac basis and fine scale wavelets. Since each entry of a Hadamard pattern $\mathbf{h}_{\mathbf{k}}$ is either -1 or +1 , one defines $\phi_{\mathbf{k}}$ as a shifted and rescaled version of $\mathbf{h}_{\mathbf{k}}$ via $\phi_{\mathbf{k}}=\left(\mathbf{h}_{\mathbf{k}}+1\right) / 2$, which can be simply encoded on the DMD. We used patterns of size $256 \times 256$ and $128 \times 128$ obtained by binning $2 \times 2$ and $4 \times 4$ groups of micromirrors. The actual pattern $\tilde{\mathbf{h}}_{\mathbf{k}}$ formed in the sample plane is in fact the convolution of the ideal pattern $\mathbf{h}_{\mathbf{k}}$ with the point spread function of the microscope $P_{\text {exc }}$ in the excitation path. Figures $\mathrm{C}$ and $\mathrm{D}$ represent WF images of a Hadamard pattern projected on a uniform and on a biological sample. A specificity of optical imaging is that the sens- ing elements $\phi_{\mathbf{k}}$ represent light intensities and are thus nonnegative which, as discussed later, has important practical implications.

Hadamard waveforms have a sort of spatial frequency (like sinusoids) which grossly depends on the typical block size of the patterns. As the power spectrum of most biological images is generally concentrated at low frequencies, the flexibility in frequency selection is important. We introduce two distinct pattern selection strategies based on the expected spatial content of the sample:

- When the sample we wish to acquire is sparse in the pixel domain as in the case of single molecule or bead imaging, no typical frequency range needs to be favored and Hadamard patterns are selected uniformly at random.

- More complex samples have a power spectrum typically decaying like a power law. This a priori information suggests that we should balance low- and high-frequency measurements in order to accurately acquire the low-frequency part of the image, which accounts for a significant part of the total variance. The half-half strategy then projects the $m / 2$ patterns with the lowest spatial frequencies to acquire a low-resolution image of the sample; the high-resolution content of the image is randomly sampled by choosing $m / 2$ measurements among the $N-m / 2$ remaining high-frequency Hadamard patterns. Such an adaptive strategy guarantees an accurate determination of the low-frequency content while allowing for the estimation of details at a finer scale.

Computational reconstruction. In CS, it is essential to enforce the sparsity of the reconstructed signal in some representation $\mathbf{W}$ that is chosen a priori. The choice of $\mathbf{W}$ highly depends on the spatial structures of the signal to be reconstructed. One would typically use a Fourier representation for oscillatory features, wavelets for pointwise singularities, curvelets for contour-like or filamentary structures [18] and so on. One could also use a concatenation of all these representations. (If one intends on using the Fourier basis as a sparsity basis, one would need to scramble the columns of the Hadamard basis since it would otherwise be coherent with sinusoids.)

After recording the fluorescence intensity during a sequence of up to 65536 consecutive patterns (Figure C), one can imagine recovering the signal $\mathbf{x}$ from these data by solving the optimization problem [3]. Because our measurements are noisy, it is actually better to relax the constraints into

$$
\min _{\mathbf{x} \in \mathbb{R}^{N}}\left\|\mathbf{W}^{T} \mathbf{x}\right\|_{\ell_{1}} \quad \text { subject to } \quad\|\mathbf{y}-\mathbf{\Phi} \mathbf{x}\|_{\ell_{2}} \leq \epsilon ;
$$

we ask that the fit holds up to the noise level. In the following, $\mathbf{W}$ will be either an orthonormal basis (e.g. Dirac basis) or an overcomplete signal representation (e.g. undecimated wavelet frame or curvelet frame). This will be clearly specified for each individual reconstruction result. For computational reasons, we find it convenient to solve a relaxed version of this problem, namely,

$$
\min _{\mathbf{x} \in \mathbb{R}^{N}}\left\|\mathbf{W}^{T} \mathbf{x}\right\|_{\ell_{1}}+\frac{\alpha}{2}\|\mathbf{y}-\mathbf{\Phi} \mathbf{x}\|_{\ell_{2}}^{2} .
$$

As is well known, there is a value $\alpha(\epsilon)$ such that the two programs coincide. For our experiments, we used the NESTA solver [30] and the regularization parameter $\alpha$ is chosen empirically depending on the noise level. When the signal is nearly sparse and the noise level low, it is known that this program finds a reconstruction with a low mean squared error (MSE).

\section{Sparse Fluorescence Images: Beads, Cells and Tissues}

Fluorescent beads. We first tested our CS microscope (with the $20 \mathrm{x}$ objective) on a sample of fluorescent beads (diameter $2 \mu \mathrm{m}$, peak emission at $520 \mathrm{~nm}$, Fluorospheres Invitrogen) deposited on a glass coverslip. At a low density of beads, the WF image is the superposition of a few fluorescence spots on a dark background, a signal similar to that of single molecule imaging data in biology [19]. As for the sparsity basis $W$, we obtained nearly equivalent results using 

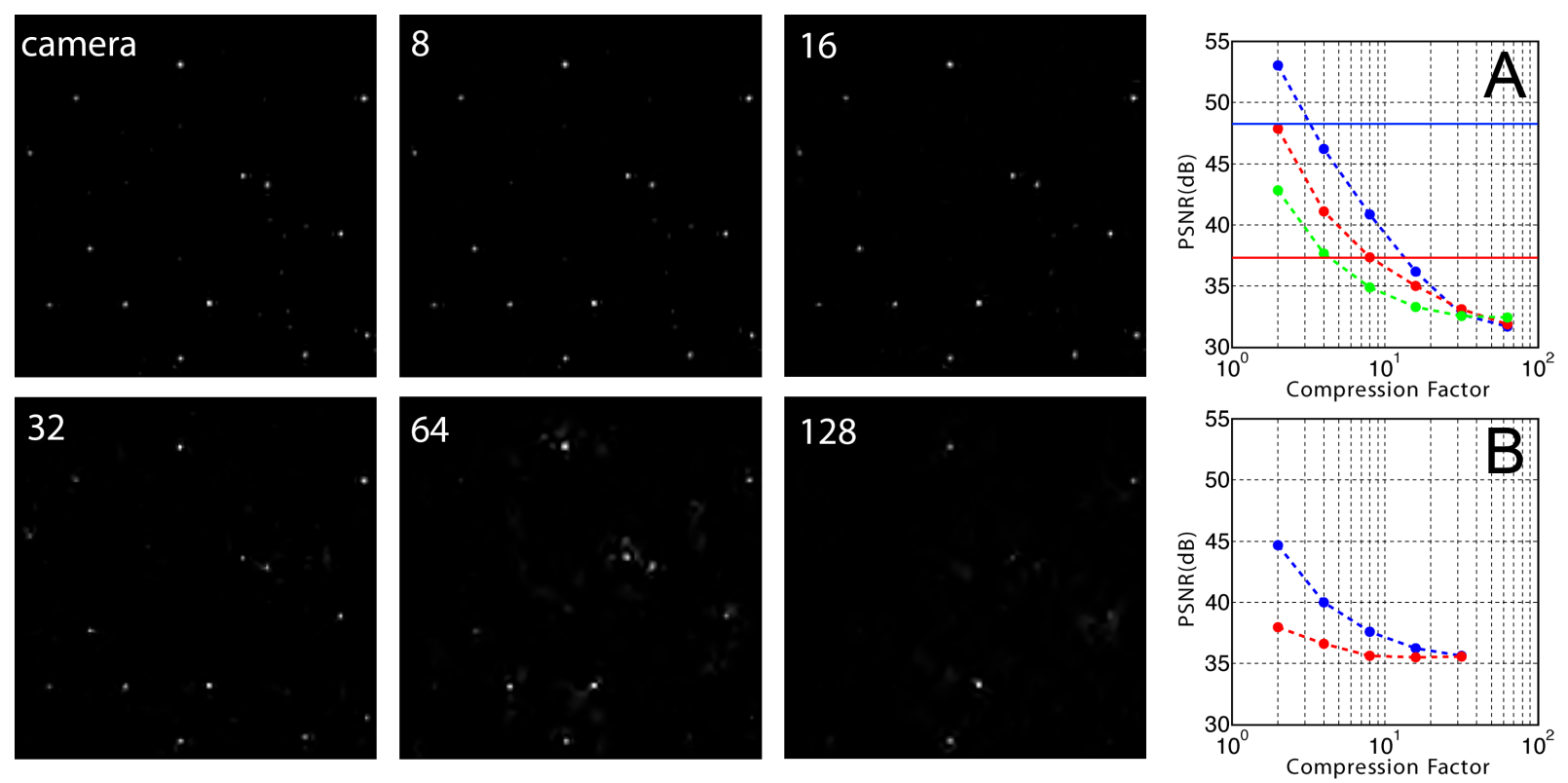

Fig. 2. Top left to bottom right: camera snapshot and reconstructed 256-by-256 bead images for values of the undersampling ratio equal to 8 , 16, 32, 64 and 128. (A) Plot of the PSNR (see text) for a nominal illumination level (blue curve) and for the same level reduced by a factor 10 (red curve) and a factor of 100 (green curve) (simulated data). The solid lines correspond to the PSNR in raster scan for the same surfacic illumination. (B) Same as (A) for the experimental data.

the Dirac basis or a wavelet transform. Here we show images reconstructed with the wavelet transform and using a number of random $256 \times 256$ Hadamard patterns decreasing from 16384 down to 512 . (To be complete, we used a weighted $\ell_{1}$ norm in eq. [6] where the weight of each coefficient is inversely proportional to scale.) In the following, the undersampling ratio is the ratio between the number $N$ of pixels and the number $M$ of measurements. As shown in Figure 2, most of the bead positions are recovered with undersampling ratios up to 64 , corresponding to $M \sim 1.5 \%$ of $N$. At higher undersampling ratios, beads with low intensities are lost.

To quantify the distorsion of the reconstructed image as a function of the undersampling ratio, we calculated the Peak Signal-toNoise Ratio, PNSR $=10 \log \left(d^{2} /\right.$ MSE $)$ where MSE $=N^{-1} \| \hat{\mathbf{x}}-$ $\mathbf{x}_{\text {ref }} \|_{2}^{2}$, the squared distance between the reconstructed image from all the $256 \times 256$ possible measurements and that which only uses a fraction. Above, $d$ is the dynamical range of the reconstruction obtained from a full sample. As shown in Figure 2A, the PSNR decreases with the undersampling ratio (blue curve) and seems to reach a plateau at ratios above 64 where most of the beads are lost. Since beads with low intensities are lost before brighter beads, we made a second set of measurements with an excitation light intensity divided by 100 to assess the effect of illumination on compression efficiency. The red curve in Figure 2A represents the PNSR of the reconstructed images as a function of the undersampling ratio. As expected, the PNSR is lower than that for the nominal illumination and reaches a plateau at an undersampling ratio of about 10 , where almost all the beads are lost. This clearly shows that the distortion of the reconstructed image is strongly affected by the amount of detected fluorescent photons. Indeed at such low intensities, photon noise (also termed, shot noise) may be significant.

To further explore the impact of photon noise on the compression efficiency, we performed numerical simulations on an artificial image of fluorescent beads made of 50 Gaussian spots (FWHM 3 pixels) randomly positioned in the field of view of size $256 \times 256$ pixels. The simulated nominal illumination intensity $I_{0}$ was set so that the resulting flux (i.e. the sum of the signals over all the pixels) was equal to $f_{0}=6.4 e 3$. Each measurement $y_{k}$ was then computed as one realization of a Poisson process with mean $\left\langle\phi_{\mathbf{k}}, \mathbf{x}\right\rangle$.
Reconstructions are processed with intensities $I_{0}, I_{0} / 10$ and $I_{0} / 100$ for a range of undersampling ratios between 2 and 64. As shown by the PSNR curves (Figure 2B), these simulations qualitatively reproduce the loss of compression efficiency for low-light levels but fail to quantitatively estimate the PSNR of the reconstructed images. This suggests that photon noise is not the only source of image degradation in our imaging system. A possible additional cause is the discrepancies between the theoretical patterns and the effective illumination profiles in the sample plane.

Lily anther slice. In order to investigate the potential of CFM for biological samples, we imaged slices of endogeneously fluorescent lily anther (Carolina Biological Supply). A conventional epifluorescence image of a slice (Excitation $488 \mathrm{~nm} /$ Emission $520 \mathrm{~nm}$ ) recorded on a CCD camera can be seen in Figure 3 (upper left). The resolution of this image has been sampled down to $128 \times 128$ pixels. We recorded the same image with the CFM setup by illuminating the sample with $16384=128^{2}$ different Hadamard patterns (complete basis). For this experiment, we used a 20x lens with a 0.75 NA. Further, we used curvelets as sparsity basis $\mathbf{W}$ since they are known to sparsely encode contour-like structures together with the half-half strategy described earlier to account for both low-spatial frequencies shapes and higher frequencies details of the sample structure. (We again used a weighted $\ell_{1}$ norm with weights inversely proportional to scale.) Reconstructed images with varying undersampling ratios from 1 to 8 are displayed in Figure 3 (top). Here, the method reconstructs images satisfyingly up to an undersampling ratio of about 8. Compared to fluorescent beads, this lower figure can be primarily attributed to the lesser sparsity.

Another important issue is that this sample is not fully two dimensional. Due to the thickness of the slice (about $50 \mu \mathrm{m}$ in this case, compared to the focal depth $\sim 1 \mu \mathrm{m}$ ), the contrast of Hadamard patterns diminishes away from the focal plane. This yields a nonmodulated background signal and, as further discussed below, the photon noise associated to this signal affects the image reconstruction by "hiding" the useful information contained in the intensity fluctuations due to the variations in the illumination patterns (Figure 1E). 
Zyxin-mEOS2 COS7 cells. In many biological applications, it is essential to use high magnification and high NA optics and we thus aimed at testing CFM in these imaging conditions (oil-immersion objective $60 x$, NA 1,45). To overcome the limitations due to the short focal depth of a high NA objective, we used photoactivation techniques. COS7 cells were transfected with Zyxin-mEOS2 [21]. Zyxin is a protein mainly expressed in the cellular focal adhesions, at the surface on which the cells are plated. It was fused to mEOS2, a genetically-encoded photoconvertible fluorescent protein tag [20] widely used in super-resolution microscopy, that has green fluorescence in its native state (Excitation 506nm / Emission 519nm) and can be converted to a red-emitting state (Excitation 573nm / Emission $584 \mathrm{~nm}$ ) upon illumination with violet light. The COS7 cells were plated at density of 100000 cell $/ \mathrm{ml}$ on $18 \mathrm{~mm}$ coverslips on a 12 well plate. The cells were transfected with Eos-Zyxin using chemical transfection (Fugene) 4-5 hours after plating and experiments were performed on live cells 18-30 hours after the transfection. By using an evanescent wave excitation with a laser at $405 \mathrm{~nm}$, we could convert proteins situated at the vicinity $(\sim 100 \mathrm{~nm})$ of the glass coverslip. Therefore, in our sample, the green emitting fluorophores are located within the $3 \mathrm{D}$ cellular volume while red-emitting proteins constitute a two-dimensional sample. The superimposed epifluorescence images in the green and red channels (256x256 pixels) are shown in Figure 3 (bottom, left). The same 2D ensemble of pho- toconverted proteins was subsequently imaged with the CFM setup with 32768 different 256x256 pixels Hadamard patterns (half of the full basis). For this set of data, the pixel size in the sample plane of each Hadamard pattern is $430 \mathrm{~nm}$, about twice the diffraction limit. As for the bead images, we used a wavelet transform as sparsity basis W.

The reconstructed images for undersampling ratios varying between 2 and 15 are displayed in the second row of Figure 3. For direct comparison with the conventional epi-fluorescence WF image, a dual color image obtained by superposition of the red converted Zyxin-mEOS2 image and the green native Zyxin-mEOS2 image is shown in Figure 3. It is noteworthy that even if the fluorescence emission of transfected COS7 cells is low compared to the lily anther slice (by about a factor of 10), the quality of the reconstructed CS images is good for undersampling ratios up to 8 and starts to be degraded at a ratio of about 15 . The very low background of our two-dimensional sample clearly enables a better reconstruction. Since fluorescent proteins are very sensitive to photobleaching, one has to use low illumination to minimize this effect during acquisition. Thus, photon noise effects, which manifested themselves only at reduced illumination intensities in the bead images, appear to be a limiting factor for CS imaging of less fluorescent and/or photo-damageable samples. The impact of photon noise on CFM is discussed in more details in a later section.
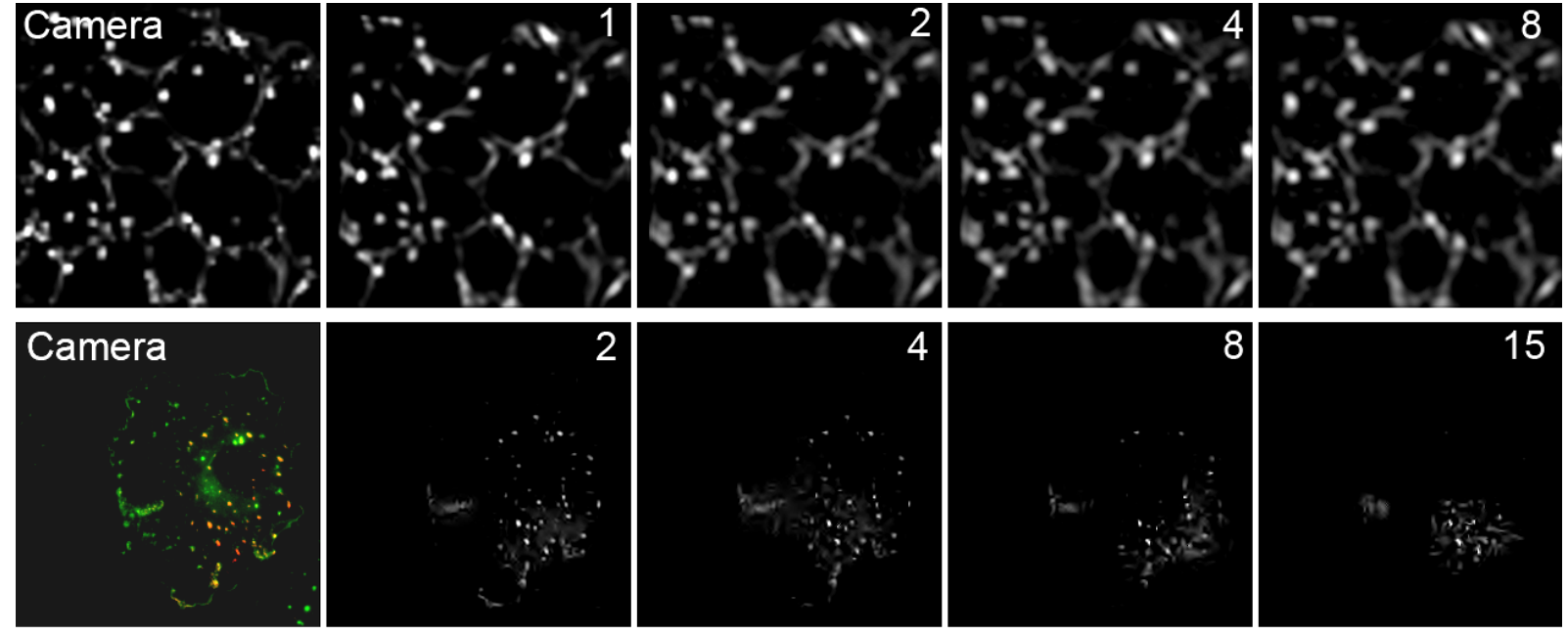

Fig. 3. Upper line: CS imaging of a slice of a lily anther. Left: Original image (128x128 pixels) by conventional epifluorescence microscopy. Left to right: the same sample imaged by CFM with undersampling ratios between 1 and 8. Lower line: CS imaging of COS7 cells expressing Zyxin-mEOS2. Left: superposition of the conventional epifluorescence images of the native (green) and converted form (red) of the markers. Left to right: CFM images of the converted form of the markers at undersampling ratios equal to $2,4,8$ and 15.

\section{Hyperspectral Imaging in CFM}

Hyperspectral imaging is defined as the combined acquisition of spatial and spectral information. In biological imaging, a growing range of applications such as the study of protein localization and interactions require quantitative approaches that analyze several distinct fluorescent molecules at the same time in the same sample [22]. These applications are in fact becoming ever more common with the avail- ability of an increasing panel of fluorescent dyes and proteins with emission ranging from the UV to the far red [23].

Multicolor data are usually acquired by selecting a few distinct spectral bands. However, in many cases, the incomplete separation of the different color channels due to the presence of autofluorescence, along with cross-excitation and emission "bleed-through" of one color channel into the others render the interpretation of multiband images difficult and/or ambiguous. To overcome these limitations, it would often be preferable to record the full spectral information at each pixel of the image. 
In this context, two elements make the potential benefits of CS particularly appealing for hyperspectral measurements. First, a full data acquisition can take up a very long time since the number of voxels $N$ quickly gets very large. Second, the signal becomes comparably sparser as the dimension increases. To demonstrate the possibility of CFM for hyperspectral fluorescent imaging, we modified the setup and replaced the point detector by a spectrometer coupled to an EMCCD camera (Evolve 512, Photometrics USA). The entire spectrum between $520 \mathrm{~nm}$ and $640 \mathrm{~nm}$ is recorded on $128 \times 1$ pixels. We spin-coated on a glass coverslip a mixture of 3 types of fluorescent beads (TransFluo Beads, Invitrogen, USA) with different emission spectra in our detection band (see Figure 4A for a gray WF image of the sample). A complete set of $256 \times 256$ Hadamard patterns was subsequently projected on the sample and, for each projected pattern, we recorded the fluorescence spectrum.

The computational reconstruction of hyperspectral data can be performed in two different manners. The simplest one is a direct extension of the monochromatic case and consists in reconstructing each spectral band independently from the others. This approach, however, does not fully account for the particular structure of the hyperspectral data. Rather, it is worthwhile to exploit sparsity in both the spatial and spectral domains. Hence, we propose a computational reconstruction by solving the same problem as in [6] with the following modification: $\mathbf{x}$ is now the full $2 \mathrm{D}-\lambda$ data cube and $\boldsymbol{\Phi}$ and $\mathbf{W}$ are waveforms $\phi_{k}[i, \lambda]$ of both space and wavelength. In these experiments, $\mathbf{W}$ was obtained by tensorizing the Dirac basis in spacewell adapted to point-wise structures like beads-with a wavelet basis along the spectral dimension which is well suited for smooth variations and occasional transients. A slice of the $2 \mathrm{D}-\lambda$ sensing matrix at a fixed $\lambda$ is the same 2D partial Hadamard transform.

We obtained full color images by pooling the data cube-see Figure 4 -into 3 spectral bands: blue $(500-530 \mathrm{~nm})$, green $(530-560$ $\mathrm{nm})$ and red (560-630 $\mathrm{nm})$. Such multicolor images are shown for varying values of the undersampling ratio in Figure 4 . We observe that almost no degradation is seen for undersampling factors up to 16. Furthermore, hyperspectral reconstructions provide the spectrum of each individual bead from the reconstructed $2 \mathrm{D}-\lambda$ cube (the normalized spectra of 3 different beads are shown in 4F). The spectra are correctly reconstructed for undersampling factors up to 64 . Interestingly, when reducing the number of measurements, the distortion primarily affects the low intensity parts of the spectra, similar to the effect of increasing undersampling on the dimmer beads in monochromatic images.

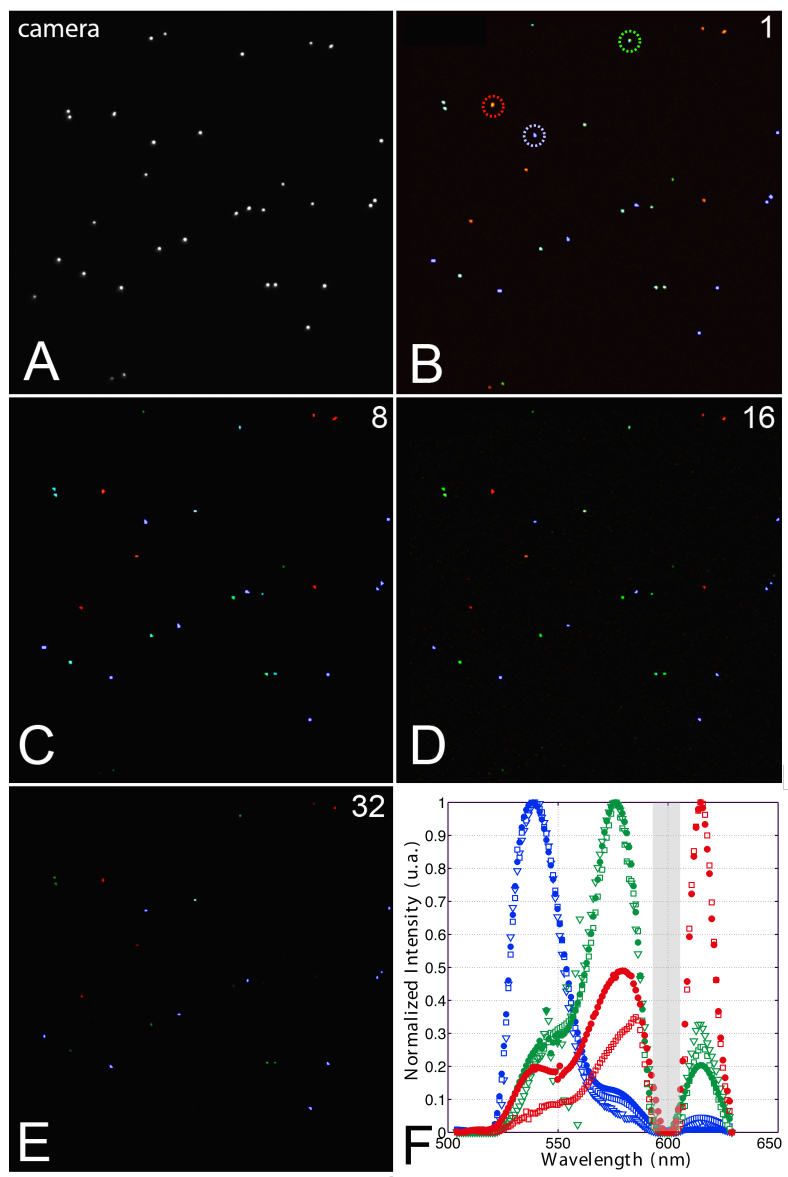

Fig. 4. (A)-(E) Camera snapshot and reconstructed 256-by-256 bead images for undersampling ratios equal to $1,8,16$ and 32. (F) Normalized spectra (128 spectral lines) of 3 individual different beads circled in (B) for undersampling ratios equal to 1 (plain circles), 32 (squares) and 64 (triangles). The grey area in the spectrum represents a rejection band of the dichroic mirror used in our setup.

\section{Discussion and Perspectives}

We have developed an imaging approach based on the concepts of CS theory which, on samples relevant for biological imaging, allows the reconstruction of fluorescence images with undersampling ratios up to 64 . While this constitutes a significant gain over undersampling ratios achieved in prior CS-based imaging approaches, several factors (computational, instrumental or noise-related) still contribute to limit the current performances of CFM. Below, we discuss them as well as the prospects for future developments and applications.

Point spread function and its modeling. In the current implementation of CFM, we neglected the role of the point spread function (PSF) of the excitation pathway (corresponding to the lenses $f_{1}, f_{2}$ and $f_{3}$ and the objective) and used the idealized matrix with zeros and ones (depending whether a pixel is illuminated or not) for the inversion. In reality, the PSF acts as a low pass filter, meaning that those patterns illuminating the sample are spatially smoother than their theoretical counterparts. In the framework of CS, it is well known that recovering a compressed signal from a sensing matrix that departs from the real one may, in general, dramatically degrade the recon- 
struction quality. In our case, however, this approximation only has minor consequences. This holds for essentially two reasons. In our experiments, the individual pixel size of the Hadamard patterns was at least twice as large as the diffraction-limit of the microscope. In other words, the PSF only has a minor filtering impact on the patterns. Second, neglecting the effect of the PSF in the reconstruction does not dramatically reduce the quality of reconstruction. To see this, let $\mathbf{f}$ be the PSF of the lens so that the collected fluorescence is of the form $y_{k}=\left\langle\phi_{\mathbf{k}} * \mathbf{f}, \mathbf{x}\right\rangle$ (we measure the dot product between the object of interest and the theoretical patterns convolved with the PSF). If the PSF is symmetric around the origin, we have

$$
\mathbf{y}=\boldsymbol{\Phi} \mathbf{F}
$$

in which $\mathbf{F}$ is the linear convolution with the PSF. Hence, neglecting the PSF recovers a signal $\tilde{\mathbf{x}}$ obeying $\mathbf{y}=\boldsymbol{\Phi} \tilde{\mathbf{x}}$; in other words, one gets $\tilde{\mathbf{x}}=\mathbf{F} \mathbf{x}$ which is $\mathbf{x}$ at the resolution of the microscope. Indeed, assuming that the lenses the excitation and emission PSFs are identical, the CSM has the same resolution as its wide-field equivalent. Now if one wishes to account for the PSF, one would need to solve a joint decompression-deconvolution problem. If the deconvolution should provide a higher resolution image, it is well-known that it is usually at the cost of noise amplification. Here again, the sparsity prior used for decompression should of course help regularizing this deconvolution step.

Noise and MSE. It is crucial to examine noise figures in our setup. There are two important facts affecting the quality of reconstruction: the noise distribution associated with CS-type data and the undersampling ratio.

We assume below that photonic noise is the limiting source of noise, an assumption which sets CS-based optical systems apart from the common theoretical CS framework [24, 10]. To understand the important trade-offs, we compare the respective situations of RS and CS, two point detection imaging techniques. Below, we put $I_{\mathrm{RS}}$ and $I_{\mathrm{CS}}$ for the excitation intensity per unit area and per unit time during RS and CS acquisition. Likewise, $T_{\mathrm{RS}}$ and $T_{\mathrm{CS}}$ are durations of excitation (for a single measurement). Finally, we set $\lambda_{\mathrm{RS}}=I_{\mathrm{RS}} \times T_{\mathrm{RS}}$ and similarly for $\lambda_{\mathrm{CS}}$. In practice, all these parameter values are adjusted according to factors that one wants to optimize (acquisition speed, sensitivity, photobleaching and so on) and they must be evaluated on a case by case basis. Hence, rather than an exhaustive comparison of the relative merits of RS and CS acquisition, the discussion below aims at providing a general framework to understand the nature of the noise in CS measurements.

In the case of RS, the $i$ th pixel measurement is distributed as a Poisson random variable with mean and variance $\lambda_{\mathrm{RS}} \times x[i]$. Using the scaled observed data yields a per-pixel MSE equal to

$$
\operatorname{MSE}(\mathrm{RS})=N^{-1} \sum_{i} \mathbb{E}(\hat{x}[i]-x[i])^{2}=\lambda_{\mathrm{RS}}^{-1} \times \bar{x},
$$

where $\bar{x}=N^{-1} \sum_{i} x[i]$. For CS, suppose first that we collect all Hadamard measurements (no undersampling). Each measurement is an independent Poisson variable with mean $\lambda_{\mathrm{CS}} \times\left\langle\phi_{\mathbf{k}}, \mathbf{x}\right\rangle$. With patterns of the form $\frac{1}{2}\left(1+\mathbf{h}_{\mathbf{k}}\right)$ where $\mathbf{h}_{\mathbf{k}}$ is a Hadamard sequence, one can decompose the mean value of $y_{k}$, as

$$
\lambda_{\mathrm{CS}} \times\left\langle\phi_{\mathbf{k}}, \mathbf{x}\right\rangle=\frac{\lambda_{\mathrm{CS}}}{2}\left[N \bar{x}+\left\langle\mathbf{h}_{\mathbf{k}}, \mathbf{x}\right\rangle\right]
$$

Hence, this is the sum of a DC offset and a Fourier-like component. The presence of the DC offset (which prevents the optical patterns from being negative) impacts the data SNR. Indeed, a possible source of concern is that for many high-frequency components, $N \bar{x}$ may be much greater than the magnitude of $\left\langle\mathbf{h}_{\mathbf{k}}, \mathbf{x}\right\rangle$ - the DC component dominates the high-frequency coefficients. Therefore, when measuring a high-frequency component, we need to deal with a large amount of noise coming from the average fluorescence of the sample under study. This situation is arguably very different than in other applications-for instance, the acquisition of radio-frequency signals-where one can use sensing waveforms that take on negative values by switching the phase of the object we wish to acquire [7]. Inverting the Hadamard matrix gives a noisy image $\hat{x}[i]$ obeying

$$
\mathbb{E} \hat{x}[i]=x[i] \quad \text { and } \quad \operatorname{Var}(\hat{x}[i])=\frac{2}{\lambda_{\mathrm{CS}}} \bar{x}
$$

(see the Appendix for details). In contrast to RS microscopy, we see that CFM yields a spatially invariant noise level in the pixel domain. In other words, by measuring the image projection on an incoherent basis, the noise gets spread equally over all the pixels. Hence, before applying any processing, the CS situation is more favorable for recovering brighter areas but less so for dimmer regions. Summing up gives

$$
\operatorname{MSE}(\mathrm{CS})=2 \lambda_{\mathrm{CS}}{ }^{-1} \times \bar{x} .
$$

This analysis also shows that it is essential to minimize the sources of signal which could contribute to a constant background and increase $\bar{x}$. This offset could be due to the nature of the sample itself but can also originate from stray light or out-of-focus fluorescence. These considerations explain, at least qualitatively, why the beads sample, where the background $\bar{x}$ is low compared to the bright spots and is purely bi-dimensional, is favorable for CFM.

To consider the effect of statistical estimation procedures or data processing, consider the beads sample again in which sparsely distributed beads are located in the field of view. Each bead is an isolated bright spot surrounded by wide non-fluorescent areas. Suppose then that we were to apply a thresholding estimator, setting to zero all intensities below a certain level, and keeping those above threshold. Then one would obtain a very low MSE in the CS setting since dark pixels would be correctly set to zero while bright pixels would have a variance that is orders of magnitude lower than that achievable in the RS case. In short, thresholding would effectively filter out the offsupport noise and the situation would be extremely favorable to the CS approach. Quantitatively, if there are $K$ bright pixels, the error after estimation for RS and CS would behave like

$$
\operatorname{MSE}(\mathrm{RS})=\lambda_{\mathrm{RS}}{ }^{-1} \times \bar{x}, \quad \operatorname{MSE}(\mathrm{CS})=2 \lambda_{\mathrm{CS}}{ }^{-1} \times \frac{K}{N} \times \bar{x} .
$$

Note the potentially enormous reduction in MSE by the factor $K / N$. Conversely, RS would be more effective for smooth and bright images (sparse images in the frequency domain).

The comparisons between RS and CS microscopy above are valid as long as the same illumination intensity per pixel is assumed and the number of measurements $M$ is equal to the number of pixels. We now discuss the effect of undersampling. Now the CS recovery is both an inversion and a denoising algorithm and the recovery error depends on the compressibility of the signal (on how sparse it is). For instance, in the fully sampled case where sparsity is assumed in the spatial domain, the CS recovery would essentially invert the Hadamard matrix and then apply soft-thresholding to the output. Now suppose for simplicity that the signal is $K$-sparse and that the number $M$ of measurements is sufficient for perfect recovery from noiseless data. Then the squared recovery error from $M$ noisy measurements as above would roughly scale like

$$
\operatorname{MSE}(\mathrm{CS})=C_{0} \times \lambda_{\mathrm{CS}}{ }^{-1} \times \frac{K}{M} \times \bar{x},
$$

where $C_{0}$ is a small numerical constant (typically on the order of unity). Hence, the main difference with the MSE available from a full sample is a loss of a factor $N / M$ - the undersampling ratioin the MSE, compare [7]. In other words, halving the number of samples-everything else, namely, intensity and duration of excitation remaining the same-doubles the MSE. The same conclusion applies for approximately sparse signals for which the variance component of the MSE dominates the squared bias. Here again, the more 
compressible the signal (the smaller $K$ ), the better the performance (e. g. the beads sample is favorable to CFM). We would like to also note that $K / M$ is still much smaller than one so that even though we are sampling less, we may still end up with a much better MSE than in RS.

To summarize, CFM is effective when: 1) the most informative parts of the sample are brighter than its mean value and 2) the sample is highly compressible. Notice that while these two requirements are sample dependent, the second gives some flexibility since one can select a representation in which a class of signals has an optimally sparse representation.

Impact of the sample thickness. One important issue in CFM has to do with the patterned illumination of thick fluorescent samples. Indeed, with a wide-field linear excitation, the illumination propagates throughout the sample and causes the entire volume to fluoresce. Since the optical transfer function of a circular aperture (such as a microscope lens) has a bandwidth that decreases with defocusing [1], the contrast of the pattern diminishes away from the focal plane. As a result, the fluorescence coming from out-of-focus planes is not modulated as a function of the patterns. In fact, this property serves as basis for optical sectioning in structured illumination microscopy (SIM) [25]. In the case of CFM, the off-focus signal contributes to an offset signal on the detector, which, as explained above, tends to significantly degrade the quality of the reconstruction. A few strategies can be considered to add sectioning capabilities to CS based imaging systems [26], [27] [15]. One recently demonstrated approach is based on the rejection of the off-focus signal, in a way similar to that of a programmable array microscope [15]. Another method is to avoid generating any off-focus signal at all. We demonstrated it for photoactivation using an evanescent-wave (Figure 3) and this can be extended to $2 \mathrm{D}$ activation within the sample volume with two-photon temporal focusing activation [28] or light sheet illumination [29]. In the long-term, an even better strategy is to illuminate with an incoherent basis of 3D patterns and, subsequently, to directly reconstruct the sample in $3 \mathrm{D}$.

\section{Conclusions and Prospect}

This paper presented the principles and implementation of compressive sensing in fluorescence microscopy together with its applications in biomedical imaging. Our approach, which is based on a patterned excitation of the sample combined with a point-detection of the emitted fluorescence, readily allows for substantial undersampling gains when compared to traditional raster-scanning approaches. It could also be useful in situations, such as a diffusing media, where direct imaging on a multi-pixel detector is not possible. Furthermore, we have set forth a distinctive prospect for hyperspectral acquisition, which has great potential for multicolor single molecule imaging. More generally, the acquisition of 3D, 4D (three spatial dimensions and one spectral or temporal dimension) or even higher dimensional signals puts unrealistic constraints on system resources. It is indeed hard to imagine that one would want to sample such huge data cubes at rates anywhere close to the Shannon rate. The key is that multidimensional signals become increasingly redundant in the sense that their information content grows at a much lower rate than the number of voxels. For example, movies are comparably far more compressible than still pictures. Likewise, hyperspectral movies are far more redundant than monochromatic movies, and so on. Expressed differently, the ratio between the number of degrees of freedom and the number of voxels decreases very rapidly as the dimension increases. The extreme sparsity of higher dimensional signals cannot be ignored and we expect the advantages of CFM to become paramount in such applications.

\section{Appendix}

This short appendix justifies our SNR calculations, and we begin by introducing some notation. We denote by $\mathbf{H}$ the Hadamard matrix and by 1 the vector with all entries equal to one. Hence, acquiring all Hadamard patterns gives us independent Poisson variables with means

$$
\mathbf{d}=\mathbf{S} \mathbf{x}:=\frac{1}{2}\left(\mathbf{1 1}^{T}+\mathbf{H}\right) \mathbf{x}
$$

Here, we set $\lambda_{\mathrm{CS}}=1$ as the general case can be obtained via a simple rescaling. Hence, our estimate is of the form

$$
\hat{\mathbf{x}}=\mathbf{S}^{-1} \mathbf{y}
$$

and it is easy to verify that

$$
\mathbf{S}^{-1}=\frac{2}{N}\left(\mathbf{H}^{T}-\frac{N}{2} \mathbf{e}_{1} \mathbf{e}_{1}^{T}\right)
$$

in which $\mathbf{e}_{1}=(1,0, \ldots, 0)$. (Observe that the first entry is a bit special here - a pixel at this location is always illuminated-and that we could always shift the Hadamard matrix as to select any special pixel.) Since $\mathbb{E} \mathbf{y}=\mathbf{d}=\mathbf{S x}$, we have $\mathbb{E} \hat{\mathbf{x}}=\mathbf{x}$. Further,

$$
\operatorname{Cov}(\hat{\mathbf{x}})=\mathbf{S}^{-1} \operatorname{Cov}(\mathbf{y}) \mathbf{S}^{-T}
$$

where $\operatorname{Cov}(\hat{\mathbf{x}})$ is the covariance matrix of the random vector $\hat{\mathbf{x}}$. Since $\operatorname{Cov}(\mathbf{y})$ is the diagonal matrix with entries $\mathbf{d}=\left(d_{1}, d_{2}, \ldots, d_{N}\right)$, we have

$$
\operatorname{Var}(\hat{x}[i])=\sum_{j=1}^{N}\left|S^{-1}[i, j]\right|^{2} d_{j}
$$

To proceed, one verifies that

$$
\frac{N}{2}\left|S^{-1}[i, j]\right|= \begin{cases}\frac{N}{2}-1, & (i, j)=(1,1), \\ 1, & (i, j) \neq(1,1),\end{cases}
$$

and that $\sum_{j=1}^{N} d_{j}=\frac{1}{2}\left(N^{2} \bar{x}+N x[1]\right)$ together with $d_{1}=N \bar{x}$. Plugging in gives

$$
\operatorname{Var}(\hat{x}[i])= \begin{cases}(N-2) \bar{x}+\frac{2}{N} x[1], & i=1, \\ 2 \bar{x}+\frac{2}{N} x[1], & i \neq 1\end{cases}
$$

(again this highlights the role played by the special pixel). Now suppose that the special pixel is chosen so that there is no probe at this location (which can always be arranged). Then we would know that $\mathbf{x}[1]=0$ (and would not bother estimating the density at that location) and for $i \neq 1$, we would have

$$
\operatorname{Var}(\hat{x}[i])=2 \bar{x}
$$

as claimed.

\section{ACKNOWLEDGMENTS.}

Deepak Nair is gratefully acknowledged for providing the Zyxin-mEOS2 transfected COS7 cells and for his careful reading of the manuscript. This work was supported by a grant from the EADS foundation. M.C. is supported by the EADS foundation and the conseil régional Aquitaine. E. C. is partially supported by NSF via grant CCF-0963835 and the 2006 Waterman Award, by AFOSR under grant FA9550-09-1-0643 and by ONR under grant N00014-09-1-0258. 
1. Mertz J (2010) Introduction to optical microscopy (Roberts and Company Publishers).

2. Brady DJ (2010) Optical Imaging and Spectroscopy (Wiley).

3. Donoho DL (2006) Compressed sensing. IEEE Trans. Inform. Theory 52:1289-1306.

4. Candes E, Romberg J, Tao T (2006) Robust uncertainty principles: exact signa reconstruction from highly incomplete frequency information. IEEE Trans. Inform. Theory 52:489-509.

5. Lustig M, Donoho D, Pauly P (2007) Sparse MRI: The application of compressed sensing for rapid MR imaging. Magnetic Resonance in Medicine 58:1182-1195.

6. Trzasko J, Manduca A, Borisch B (2009) Highly undersampled magnetic resonance image reconstruction via homotopic $\ell_{0}$ minimization. IEEE Trans. Medical Imaging 28:106-121.

7. Yoo J, Becker S, Monge M, Loh M, Candes E, Emami-Neyestanak A (2011). Design and implementation of a fully integrated compressed-sensing signal acquisition system. Submitted to IEEE Intl. Conf. Acoustics, Speech and Signal Processing (ICASSP).

8. Mishali M, Eldar YC, Dounaevsky O, Shoshan E (2011) Xampling: Analog to digital at sub-Nyquist rates. IET Cir. Dev. and Systems 5:8-20.

9. Bobin J, Starck JL, Ottensamer R (2008) Compressed Sensing in Astronomy. IEEE Journal of Selected Topics in Signal Processing 2:718-726.

10. Duarte MF, Davenport MA, Takhar D, Laska JN, Sun T, Kelly KF, araniuk RG (2008) Single-Pixel Imaging via Compressive Sampling. IEEE Signal Process. Mag. 25:83-91.

11. Pitsianis NP, Brady DJ, Portnoy A, Sun X, Suleski T, Fiddy MA, Feldman MR, Tekolste RD (2006) Compressive imaging sensors. Proceedings of SPIE - The International Society for Optical Engineering Vol. 6232.

12. Gazit S, Szameit A, Eldar YC, Segev M (2009) Super-resolution and reconstruction of sparse sub-wavelength images. Optics Express 17:23920.

13. Brady DJ, Choi K, Marks DL, Horisaki R, Lim S (2009) Compressive holography. Optics Express 17:13040-13049.

14. Katz O, Bromberg Y, Silberberg Y (2009) Compressive ghost imaging. Appl. Phys Lett. 95:131110.

15. Wu Y, Ye P, Mirza IO, Arce GR, Prather DW (2010) Experimental demonstration of an optical-sectioning compressive sensing microscope (CSM). Opt. Express 18:2456524578.

16. Candes E, Romberg J (2007) Sparsity and incoherence compressive sampling. In verse problems 23:969-985.
17. Candes E, Plan Y (2011) A Probabilistic and RIPless theory of compressed sensing IEEE Trans. Inform. Theory 57:7235-7254.

18. Candes E, Donoho $D$ (2004) New tight frames of curvelets and optimal representations of objects with piecewise $C^{2}$ singularities. Comm. Pure Appl. Math. 57:219-266.

19. Lord SJ, Lee HL, Moerner WE (2010) Single-molecule spectroscopy and imaging of biomolecules in living cells. Anal Chem. 82:2192-2203.

20. Wiedenmann J, Ivanchenko S, Oswald F, Schmitt F, Rocker C, Salih A, Spindler KD Nienhaus GU (2004). EosFP, a fluorescent marker protein with UV-inducible green-tored fluorescence conversion. Proc Natl Acad Sci USA 101:15905-15910.

21. Shroff H, Galbraith CG, Galbraith JA, White H, Gillette J, Olenych S, Davidson MW, Betzig $E$ (2007) Dual-color superresolution imaging of genetically expressed probes within individual adhesion complexes. Proc Natl Acad Sci USA 104:20308-20313.

22. Zimmermann T, Rietdorf J, Pepperkok $R$ (2003) Spectral imaging and its applications in live cell microscopy. FEBS Letters 546:87-92.

23. Giepmans BN, Adams SR, Ellisman MH, Tsien RY (2006) The fluorescent toolbox for assessing protein location and function. Science 312:217-224.

24. Willett R, Marcia R, Nichols J (2011) Compressed sensing for practical optical systems: a tutorial. Optical Engineering 50, 072601 1-13.

25. Mertz J (2011) Optical sectioning microscopy with planar or structured illumination Nat. Methods 8:811-819.

26. Ye P, Paredes JL, Wu Y, Chen C, Arce GR, Prather DW (2009) Compressive confocal microscopy: 3D reconstruction algorithms. Proceedings of SPIE - Emerging Digita Micromirror Device Based Systems and Applications Vol. 7210.

27. Ye P, Paredes JL, Arce GR, Wu Y, Chen C, Prather DW (2009) Compressive confocal microscopy. Proceedings - IEEE ICASSP 2009.

28. Vaziri A, Tang J, Shroff H, Shank CV (2008) Multilayer three-dimensional super resolution imaging of thick biological samples. Proc. Natl. Acad Sci. USA 105:2022120226.

29. Huisken J, Swoger J, Del Bene F, Wittbrodt J, Stelzer EH (2004) Optical sectioning deep inside live embryos by selective plane illumination microscopy. Science 305:1007-1009.

30. Becker S, Bobin J, Candes E (2011) NESTA: a fast and accurate first-order method for sparse recovery SIAM J. Imaging Sci. 4:1-39. 